\title{
True left bundle branch block and long-term mortality in cardiac resynchronisation therapy patients
}

\author{
Roksana Kisiel ${ }^{1}$, Kamil Fijorek ${ }^{2}$, Paweł Moskal ${ }^{1}$, Piotr Kukla ${ }^{3}$, Christopher Pavlinec ${ }^{4}$, \\ Jakub Stec ${ }^{1}$, Danuta Czarnecka ${ }^{1}$, Marek Jastrzębski ${ }^{1}$ \\ ${ }^{1} 1^{\text {st }}$ Department of Cardiology, Interventional Electrocardiology, and Hypertension, Jagiellonian University Medical College, Krakow, Poland \\ 2Department of Cardiology, Henryk Klimontowicz Specialist Hospital, Gorlice, Poland \\ ${ }^{3}$ Department of Statistics, Krakow University of Economics, Krakow, Poland \\ ${ }^{4}$ International PhD Programme in Medical Science, Faculty of Medicine, Jagiellonian University Medical College, Krakow, Poland
}

\begin{abstract}
A bstract
Background: Left bundle branch block (LBBB) is an important qualification criterion and determinant of prognosis in cardiac resynchronisation therapy $(\mathrm{CRT})$ patients.

Aim: Our goal was to investigate the long-term mortality and morbidity in a sizable cohort of patients with CRT with regard to the new strict LBBB definition proposed by Perrin.

Methods: We performed a longitudinal cohort study that included consecutive CRT patients. Primary endpoint (all-cause death) and secondary endpoint (all-cause death and hospitalisation for heart failure) were analysed. All preimplantation electrocardiograms were categorised as LBBB or non-LBBB according to the new definitions/criteria analysed.

Results: The survival analysis comprised 552 patients with CRT. The Perrin criteria, CRT guidelines class I indication criteria, and Strauss criteria were fulfilled in $38.9 \%, 79.4 \%$, and $62.3 \%$ of all LBBB patients, respectively. During the nine-year study period, 232 patients died and the combined endpoint was met by 292 patients. The Perrin "true LBBB" definition criteria were inferior to the Strauss "complete" LBBB definition criteria in predicting survival as reflected by Kaplan-Meier survival curves (C-statistics). Multivariate Cox regression models showed that both LBBB definitions predicted mortality, however, the Perrin definition had a higher hazard ratio (HR 0.67) compared to the Strauss definition (HR 0.51).

Conclusions: It seems that the Perrin "true LBBB" criteria are not well-suited for the selection of CRT candidates. Perhaps they do not reflect the presence of a true/complete LBBB or exclude too many patients who, despite some residual conduction in the left bundle branch, responded well to CRT.
\end{abstract}

Key words: cardiac resynchronisation therapy, complete block, criteria, left bundle branch block, mortality

Kardiol Pol 2019; 77, 3: 371-379

\section{INTRODUCTION}

Cardiac resynchronisation therapy (CRT) improves heart failure (HF)-related symptoms and long-term outcome in chronic HF patients, mainly by correcting the delayed contraction of the lateral segments of the left ventricle induced by left bundle branch block (LBBB). Several clinical echocardiographic and electrocardiographic parameters predict the long-term outcome in CRT [1-6]. The prognostic importance of LBBB in patients scheduled for CRT has been especially well-studied in the past $[7,8]$. However, conflicting results have made clinical recommendations uncertain. In some studies, patients with an LBBB morphology had better outcomes than patients with a non-LBBB morphology [8-10]. In other studies, no clinical difference or even a worse clinical prognosis was seen in patients with LBBB $[11,12]$. However, in CRT studies, several substantially different electrocardiographic criteria for LBBB have been used. We and others have shown the importance of LBBB definition/criteria in predicting long-term mortality and 

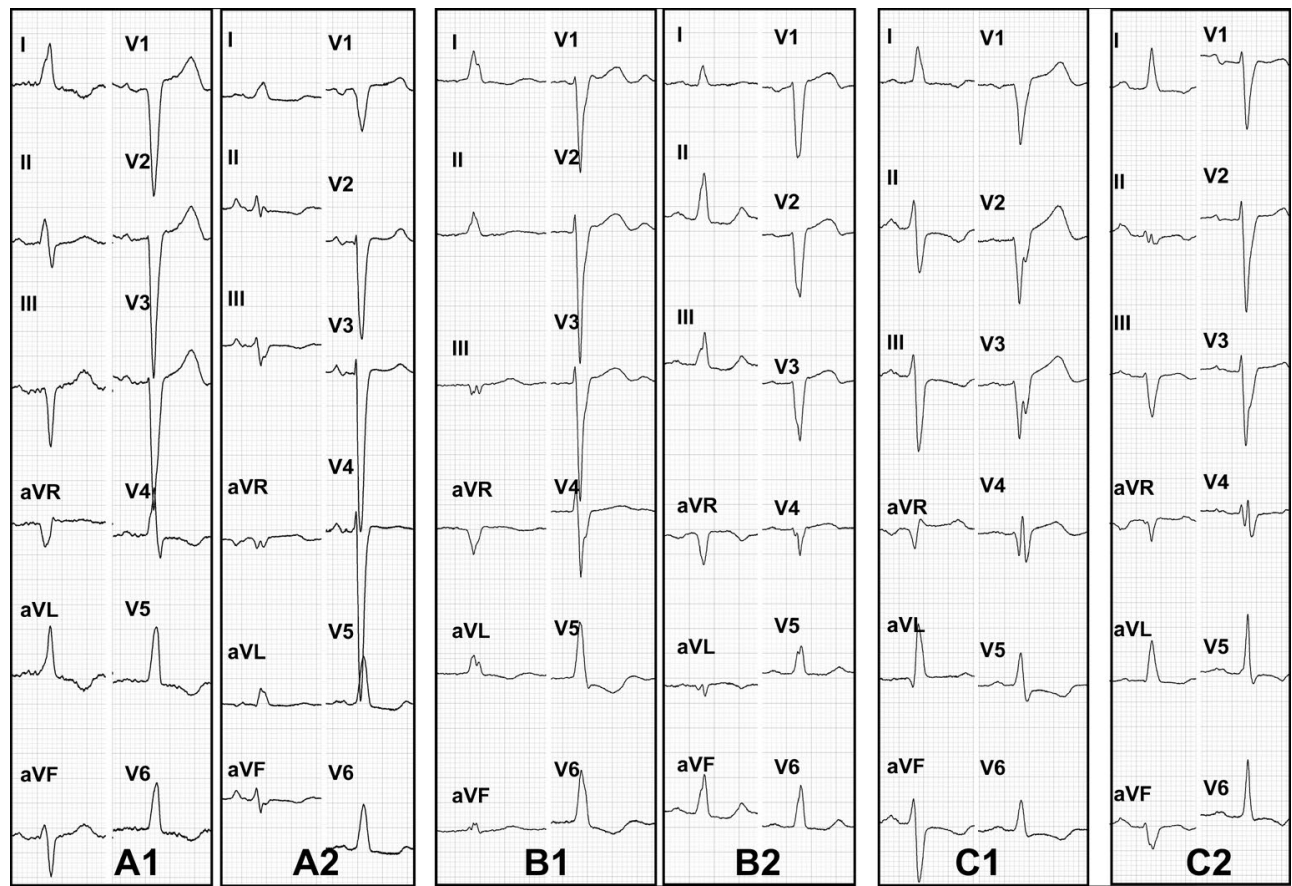

Figure 1. Examples of 12-lead electrocardiograms (ESCS) that fulfil different left bundle branch block (LBBB) criteria. A1, A2. Two examples of LBBB according to Padanilam/Perrin but not according to Strauss (only a single QRS with slur/notch) and not fulfilling the European Society of Cardiology (ESC) guidelines class I indications for cardiac resynchronisation therapy (CRT) (QRS < $150 \mathrm{~ms}) ;$ B1. LBBB according to Strauss definition and fulfilling the ESC CRT guidelines class I criteria but a non-LBBB morphology according to Perrin ( $r$ in $V_{1} \geq 1 \mathrm{~mm}$ ); B2. LBBB according to Strauss definition but neither fulfilling the ESC CRT guidelines class I criteria (QRS $<150 \mathrm{~ms}$ ) nor the LBBB criteria according to Perrin ( $r$ in $\mathrm{V}_{1}$ and $\mathrm{q}$ in $\mathrm{aVL} \geq 1 \mathrm{~mm}$ and no R/qR in $\mathrm{aVL}$ ); C1, C2. ECGs indicating LBBB according to the ESC CRT guidelines class I/level A indications for CRT but categorised as non-LBBB according to both Perrin ( $q$ in $\mathrm{aVL} \geq 1 \mathrm{~mm}$ in $\mathrm{C} 1$ and $r$ in $V_{1} \geq 1 \mathrm{~mm}$ in $\mathrm{C2}$ ) and Strauss LBBB definitions (lack of two QRS complexes in $\mathrm{I}, \mathrm{aVL}, \mathrm{V}_{1}-\mathrm{V}_{2}, \mathrm{~V}_{5}-\mathrm{V}_{6}$ with a notch/slur)

morbidity in CRT patients, which might explain the divergent results of the clinical studies regarding the impact of $L B B B$ on CRT outcome and help to formulate an optimal LBBB definition for CRT candidate selection [13, 14].

Recently, two new definitions were proposed with the intention to identify "complete" or "true" LBBB - theoretically, an optimal substrate for cardiac resynchronisation. Strauss et al. $[15,16]$, on the basis of pathophysiological data, proposed that the "complete LBBB" definition should include a longer QRS duration (> $130 \mathrm{~ms}$ in women and > $140 \mathrm{~ms}$ in men) and the presence of at least two mid-QRS notches/slurs in leads $\mathrm{I}$, aVL, $\mathrm{V}_{1}, \mathrm{~V}_{2}, \mathrm{~V}_{5}$, or $\mathrm{V}_{6}$. Padanilam et al. [17] analysed the QRS morphology of patients with electrophysiologically-confirmed total LBBB without any residual conduction and concluded that an $\mathrm{R}$ wave $\geq 1 \mathrm{~mm}$ in $\mathrm{V}_{1}$ points to an incomplete LBBB. On the basis of that study, Perrin et al. [18] proposed a new "true LBBB" definition that included the obligatory lack of hypothetical markers of residual left to right conduction, i.e. no $R$ wave $\geq 1 \mathrm{~mm}$ in $V_{1}$ and no $Q$ wave $\geq 1 \mathrm{~mm}$ in aVL. Examples of QRS morphologies that fit these two new LBBB definitions are presented in Figure 1. The Strauss definition was clinically validated by two large studies (Jastrzębski et al. [13] and Caputo et al. [14]) which showed better long-term survival of CRT patients when the "complete LBBB" definition was fulfilled. Other studies which assessed "soft" endpoints, such as better echocardiographic or clinical response [19, 20], also supported the Strauss definition as being more suitable for LBBB diagnosis in CRT candidates. In contrast, the "true LBBB" definition by Perrin et al. [18] was validated only by the PREDICT study sub-analysis, which was limited to the assessment of short-term echocardiographic response. Neither long-term mortality/morbidity analysis nor "head-to-head" comparison with the other new stricter LBBB definition is available for the Padanilam/Perrin criteria.

Our aim was to assess, in a sizable cohort of CRT patients, the "true LBBB" definition as proposed by Perrin, with regard to the long-term mortality and morbidity, and run a comparison to the Strauss LBBB definition and to the QRS morphology compliant with the current class I CRT indications according to the recognised guidelines.

\section{METHODS}

\section{Study group and outcome endpoints}

Patients who received CRT at our institution in the period from mid-2006 to the end of 2014 were included in this retrospective analysis. Relevant clinical data and pertinent procedural 
Table 1. Criteria included in the two new definitions of left bundle branch block (LBBB) and the European Society of Cardiology (ECS) class I indications for cardiac resynchronisation therapy (CRT)

\begin{tabular}{|c|c|c|c|}
\hline & $\begin{array}{l}\text { Perrin: } \\
\text { "True LBBB" }\end{array}$ & $\begin{array}{l}\text { Strauss: } \\
\text { "Complete LBBB" }\end{array}$ & $\begin{array}{c}\text { ESC CRT guidelines } \\
\text { Class I }\end{array}$ \\
\hline $\mathrm{QRS} \geq 120 \mathrm{~ms}$ & + & & \\
\hline \multicolumn{4}{|l|}{ QRS $\geq 130$ ms (female) } \\
\hline $\mathrm{QRS} \geq 140 \mathrm{~ms}$ (male) & & + & \\
\hline $\mathrm{QRS} \geq 150 \mathrm{~ms}$ & & & + \\
\hline $\mathrm{R}$ peak $>60 \mathrm{~ms}$ in $\mathrm{V}_{5} \mathrm{~N}_{6}$ & + & & + \\
\hline rS or QS in $V_{1} N_{2}$ (with R peak time $<60 \mathrm{~ms}$ ) & + & + & + \\
\hline Monophasic R in I & + & & \\
\hline Monophasic R or qR in aVL & + & & \\
\hline Notched or slurred $R$ in $\geq 1$ lead: I, $a V L, V_{5}, V_{6}{ }^{*}$ & + & & \\
\hline Mid-QRS notch or slur in $\geq 2$ leads: $I, a V L, V_{1}, V_{2}, V_{5}, V_{6}$ & & + & \\
\hline No $Q$ wave in $I, V_{5}, V_{6}$ & + & & \\
\hline No $\mathrm{Q}$ wave $\geq 1 \mathrm{~mm}$ in aVL and no $\mathrm{R}$ wave $\geq 1 \mathrm{~mm}$ in $\mathrm{V}_{1}$ & + & & \\
\hline
\end{tabular}

*The notch/slur does not have to be "mid-QRS"

data were obtained from patient medical records. Endpoint data (deaths and hospitalisations) were obtained during outpatient follow-up visits (most of our patients routinely visit our clinic for diagnostic check-ups) or telephone interviews with patients or their family members. If the patients could not be contacted (directly or indirectly), the national PESEL registry was used to check the dead or alive status. Two endpoints were analysed: primary — all-cause mortality/heart transplantation and secondary - HF hospitalisation or death from any cause.

\section{Electrocardiographic data}

Continuous 12-lead preimplantation electrocardiograms (ECGs) were recorded on an electrophysiological system (BARD LabSystems Pro, Boston Scientific, Boston, MA, USA) in all patients undergoing CRT device implantation. Both QRS duration and $R$ wave peak delay in $V_{6}$ were measured using a global method. $\mathrm{R}$ wave peak delay was measured from the earliest onset of QRS in all 12 leads on a standard ECG to the $R$ wave peak in $V_{6}$, as recommended by the American Heart Association (AHA) and the World Health Organisation (WHO) $[21,22]$. ECG tracings were analysed by two physicians blinded to the outcome of the study according to three different criteria sets: the Strauss LBBB definition, the Perrin definition, and the European Society of Cardiology (ESC) guidelines class $1 /$ level of evidence $A$ indications for CRT [23]. The Strauss LBBB definition was based on the original publication with a later modification which removed the requirement that the leads with notch/slur be "contiguous." The Perrin definition, as provided in the original publication, was based on the AHA LBBB definition with the added extra Padanilam/Perrin criteria. The ECS guidelines do not specify any LBBB definition; therefore, the guideline criteria in this study were based on the conventional LBBB definition based on $\mathrm{V}_{1}$ and $\mathrm{V}_{6}$ morphology with an added requirement of $\mathrm{QRS}$ duration $>150 \mathrm{~ms}$. The three sets of criteria/definitions are summarised in Table 1.

Any disparity in classifying patients as having LBBB or non-LBBB morphology were solved by reaching consensus between the two researchers or through consulting a third investigator when necessary. Measurements were taken using precise interval measurements and morphology assessment on the electrophysiological system with high signal augmentation and a sweep speed of $100 \mathrm{~mm} / \mathrm{s}$.

A non-specific intraventricular conduction delay (NIVCD) was diagnosed when the QRS was $\geq 120 \mathrm{~ms}$ and the criteria for diagnosis of LBBB or right bundle branch block were not met.

\section{Statistical analysis}

Continuous variables are presented as mean and standard deviation while categorical variables are presented as number and percentage. Kaplan-Meier curves were generated to trace the survival of each study endpoint. The discriminative power of a single LBBB definition was obtained using Harrell's C-statistic. C-statistic values were compared in order to determine which LBBB definition was better at predicting survival. Cox proportional hazards $(\mathrm{CPH})$ models were then used to determine the impact of LBBB on survival after adjusting for potential confounders. Variables that were deemed to have clinical relevance were preselected and entered into the multivariate $\mathrm{CPH}$ models. Results of these models are presented as hazard ratios (HRs) and 95\% confidence intervals (Cls). The proportionality assumption required for the $\mathrm{CPH}$ method was met. All statistical analyses were performed using 


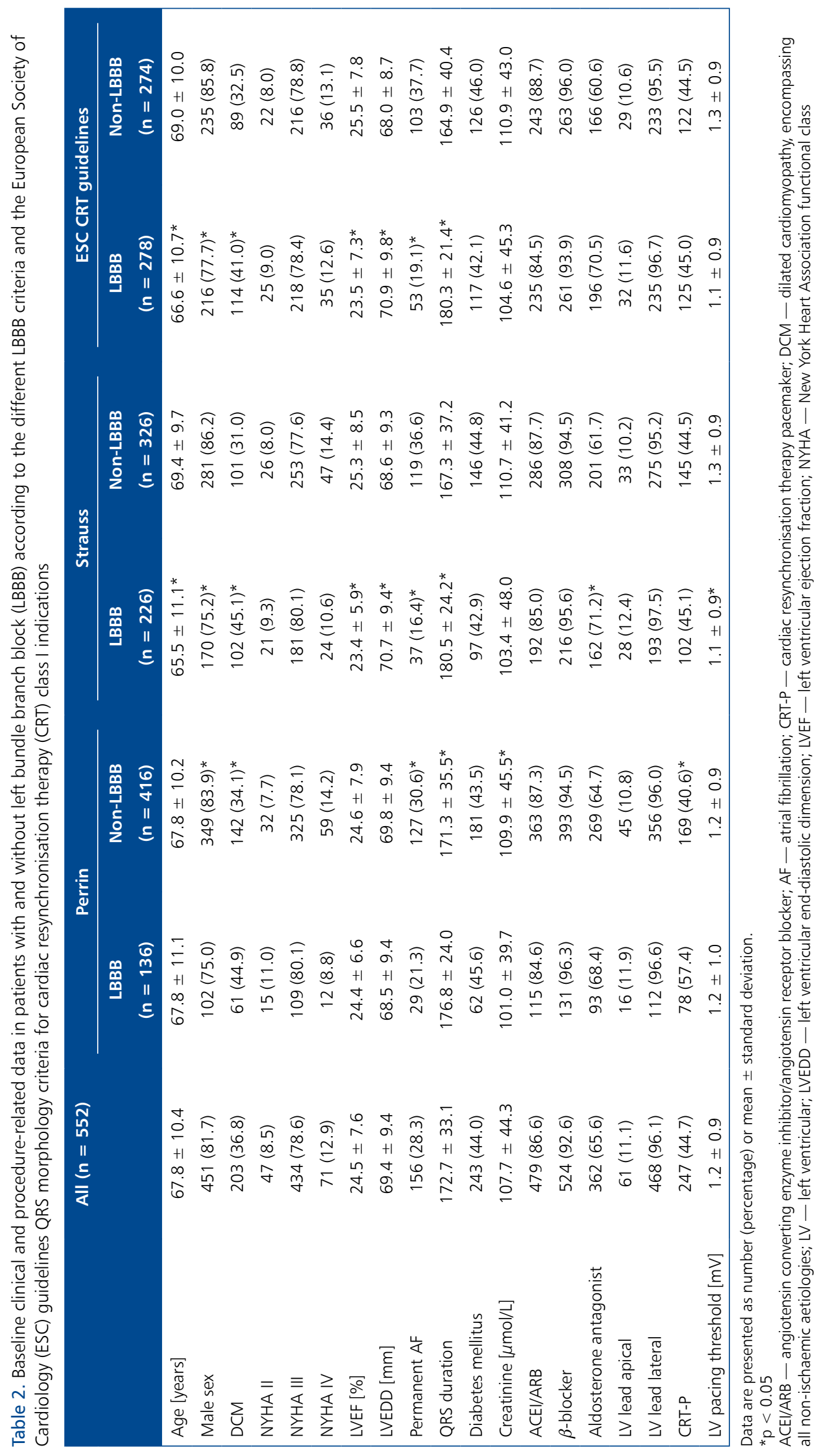


the R 3.2 software (the R Foundation for Statistical Computing, Vienna, Austria) with $\mathrm{p}$-values $<0.05$ considered statistically significant.

\section{RESULTS}

Population and implant results

The inclusion criteria were met by 590 patients who underwent CRT device implantation during the previously specified study period. Of these, 38 patients were excluded: 15 due to an unsuccessful left ventricular (LV) lead implantation (a success rate of $97.5 \%$ ), eight due to incomplete medical records, five due to a late LV lead repositioning/loss of CRT, seven due to a necessary upgrade to triple-site pacing (two LV leads), and three due to the usage of a non-standard resynchronisation approach (i.e. direct His bundle pacing, dual-site right ventricular pacing). As a result, 552 patients were left in the dataset. Baseline clinical characteristics, the presence or absence of LBBB, and the outcomes of CRT device implantation are presented in Table 2.

\section{ECG categorisation according to $\mathrm{LBBB}$ definitions}

According to the conventional definition of LBBB ( $\mathrm{rS} / \mathrm{QS}$ morphology in $\mathrm{V}_{1}$ and $\mathrm{R}$ peak in $\mathrm{V}_{6} \geq 60 \mathrm{~ms}, \mathrm{QRS} \geq 120 \mathrm{~ms}$ ), $350(63.4 \%)$ patients had LBBB, 52 (9.4\%) had NIVCD, 31 (5.6\%) had right bundle branch block, 98 (17.7\%) had paced QRS, and 21 (3.8\%) had a narrow QRS. The Perrin and Strauss definitions and the CRT ESC guideline criteria were fulfilled in 136 (24.6\%), 218 (39.5\%), and 278 (50.4\%) of all patients, respectively, and in $38.9 \%, 62.3 \%$, and $79.4 \%$ of patients with LBBB according to the conventional definition.

During the nine-year investigatory period, 232 patients met the primary endpoint of death from any cause $(n=228)$ or urgent heart transplantation $(n=4)$. The survival rates at the end of years one to seven were $89.7 \%, 80.7 \%, 70.6 \%$, $63.6 \%, 57.2 \%, 52.7 \%$, and $46.9 \%$, respectively. During this same period, 128 patients were hospitalised for an exacerbation of HF symptoms and 68 of these patients eventually died. Consequently, 292 patients met the combined secondary endpoint of all-cause mortality or hospitalisation for HF (232 patients who died and 60 patients who had HF exacerbations but survived).

Kaplan-Meier curves for the two study endpoints with regard to the Perrin definition, CRT guidelines, and Strauss criteria are presented in Figure 2. C-statistics for the Perrin definition, CRT guidelines criteria, and Strauss criteria for all-cause mortality were $0.547(p=0.004), 0.542(p=0.036)$, and 0.581 ( $p<0.001$ ), respectively, and for the secondary combined endpoint of all-cause mortality and HF hospitalisation they were 0.557 ( $p<0.001), 0.553$ ( $p=0.003)$, and 0.581 ( $p<0.001)$, respectively. The comparison of C-statistics showed that the Padanilam/Perrin definition was inferior to the Strauss definition for predicting all-cause mortality $(p=0.039)$ but not for predicting the secondary combined endpoint of all-cause mortality and HF hospitalisation ( $p=0.101$ ); no differences in C-statistics between Perrin and ESC CRT guidelines class I criteria were present for the primary $(p=0.745)$ or secondary endpoint $(p=0.985)$.

Multivariate Cox HRs for the three LBBB definitions/criteria, including other pertinent clinical variables, are presented in Tables 3 and 4. The presence of LBBB according to the Perrin definition was associated with all-cause mortality and the combined end-point of all-cause mortality and hospitalisation for HF. Prognostic value of the Perrin definition was similar to the prognostic value of the ECS CRT guidelines criteria and lower than the prognostic importance of the Strauss definition.

\section{DISCUSSION}

To our best knowledge, this is the largest cohort study with the longest follow-up to date that examines the new "true LBBB" definition proposed by Perrin et al. [18]. The major finding of the current study is that the Perrin definition seems less useful for the prediction of outcome in CRT patients. Firstly, the Perrin definition was much more exclusive because it was fulfilled by only $38.9 \%$ of all LBBB patients, whereas the Strauss definition and ESC CRT guidelines class I QRS morphology criteria were met by $62.3 \%$ and $79.4 \%$ of patients, respectively. This is an unfavourable characteristic for the potential use for CRT patient selection. Based on the known non-responder rate of approximately 30\%, it can be speculated that an optimal LBBB definition should not exclude more than $30 \%$ of patients with LBBB morphology. Secondly, the absolute difference in all-cause mortality at six years between patients with and without LBBB according to this definition was $11 \%$, which was much less than the $20 \%$ difference seen when the Strauss definition was applied. This was also reflected by the significant difference in C-statistics between these two LBBB definitions. Results for the combined endpoint of all-cause mortality and HF hospitalisations were very similar.

A possible explanation for the observed findings is that the $R$ wave $\geq 1 \mathrm{~mm}$ in $\mathrm{V}_{1}$ is, after all, not a marker for left-to-right ventricular septum activation that indicates residual conduction over the left bundle branch. Wellens [24] suggests that larger $\mathrm{R}$ waves in $\mathrm{V}_{1}$ might result from unopposed activation of the free wall of the right ventricle, which produces an activation vector directed at lead $V_{1}$. Such a situation takes place in patients with LBBB and after a large anteroseptal myocardial infarction that causes the absence or diminished right-to-left activation of the interventricular septum.

Another possible explanation is that the presence of residual $\mathrm{LBBB}$ conduction is not important from the perspective of mechanistic paradigm behind CRT. Probably, when the Strauss LBBB features are present in the ECG, the delay in the left bundle conduction must be significant enough and residual conduction might not play any important role in alleviating the delay of the activation of the lateral LV wall. Moreover, an $\mathrm{R}$ wave $\geq 1 \mathrm{~mm}$ in $\mathrm{V}_{1}$ and/or $\mathrm{Q} \geq 1 \mathrm{~mm}$ in $\mathrm{aVL}$ 


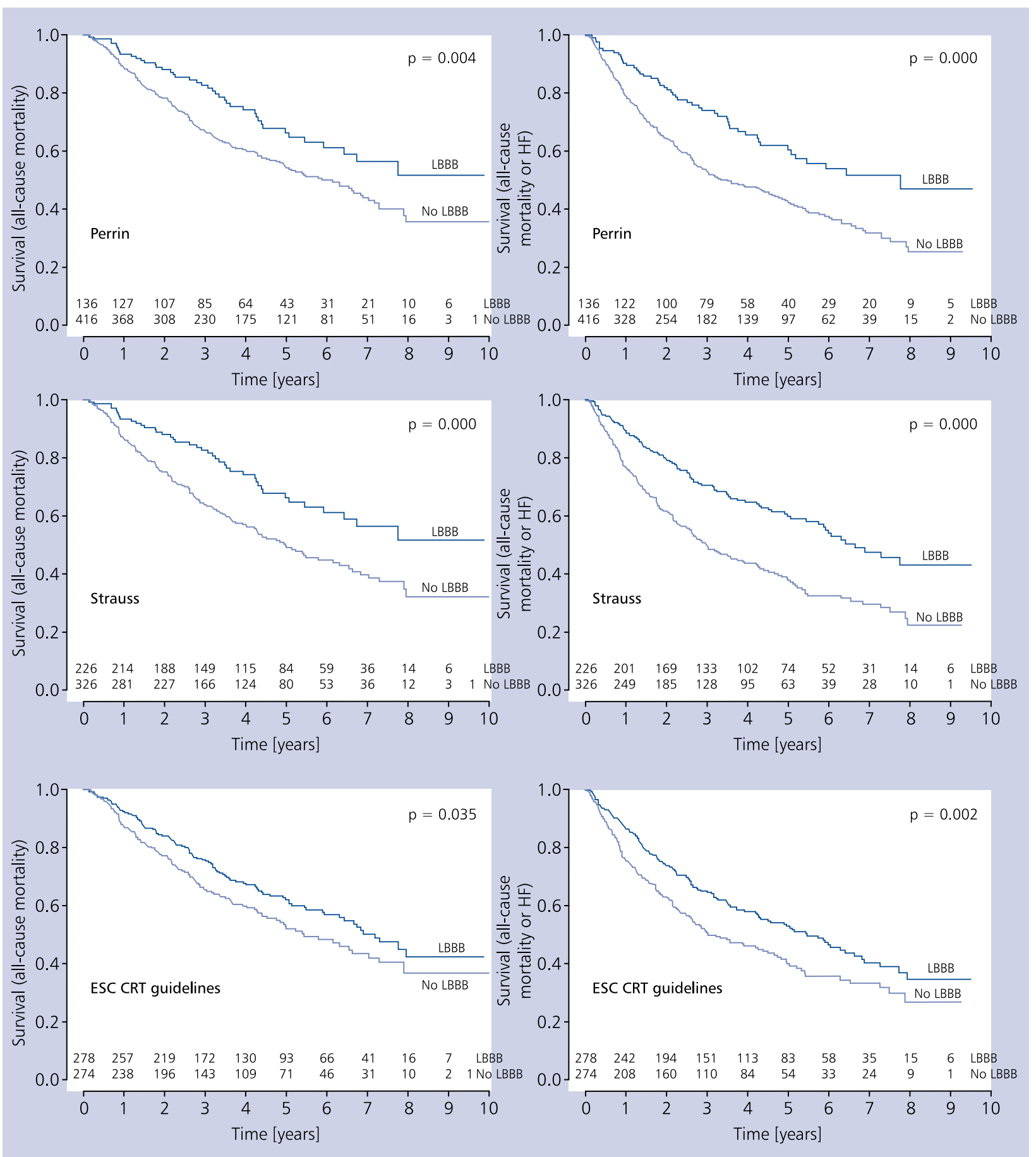

Figure 2. Kaplan-Meier survival curves for two endpoints: all-cause mortality and the combination of all-cause mortality and hospitalisation for heart failure (HF) with regard to the two different definitions of left bundle branch block (LBBB; Perrin and Strauss) and the European Society of Cardiology (ESC) guidelines class I (level of evidence A) for cardiac resynchronisation therapy

might be related to the residual conduction in the left septal fascicle only (no conduction in the left anterior and posterior fascicles of the left bundle branch). Such a situation probably results in the asynchronous activation of the $\mathrm{LV}$, similar to the activation seen in complete LBBB (when LV is also activated from the interventricular septum via breakthrough points from the right ventricular depolarisation). Therefore, despite some residual left bundle conduction, the asynchrony might be large enough to warrant CRT and relate to a better prognosis post CRT. If this is the case, then the exclusion of 
Table 3. Multivariate Cox regression models for all-cause mortality in patients with and without left bundle branch block (LBBB) according to the Perrin and Strauss definitions and the European Society of Cardiology (ESC) guidelines QRS morphology criteria for cardiac resynchronisation therapy (CRT) class I indications with regard to potential confounding variables

\begin{tabular}{|c|c|c|c|}
\hline & \multicolumn{3}{|c|}{ HR $(95 \% \mathrm{Cl})$} \\
\hline & Perrin & Strauss & ESC CRT guidelines \\
\hline LBBB & $0.67 *(0.48-0.95)$ & $0.51 * *(0.38-0.69)$ & $0.73 *(0.56-0.96)$ \\
\hline Age $60-70$ years & $1.02(0.70-1.50)$ & $0.88(0.59-1.29)$ & $0.99(0.68-1.45)$ \\
\hline Age $>70$ years & $1.36(0.94-1.97)$ & $1.20(0.82-1.74)$ & $1.31(0.90-1.90)$ \\
\hline Male sex & $1.05(0.72-1.53)$ & $1.04(0.71-1.52)$ & $1.05(0.72-1.54)$ \\
\hline Ischaemic aetiology & $1.34 *(0.99-1.82)$ & $1.31(0.96-1.77)$ & $1.35(1.00-1.83)$ \\
\hline NYHA III & $1.82(0.89-3.72)$ & $1.90(0.93-3.90)$ & $1.88(0.92-3.84)$ \\
\hline NYHA IV & $4.30 * *(2.00-9.21)$ & $4.63^{* *}(2.16-9.92)$ & $4.54^{* *}(2.12-9.72)$ \\
\hline Permanent AF & $0.83(0.61-1.12)$ & $0.73 *(0.54-1.00)$ & $0.78(0.57-1.07)$ \\
\hline LVEF $20 \%-30 \%$ & $0.75(0.56-1.01)$ & $0.75(0.55-1.01)$ & $0.76(0.56-1.02)$ \\
\hline LVEF $>30 \%$ & $0.61 *(0.40-0.95)$ & $0.56 *(0.36-0.87)$ & $0.58 *(0.38-0.90)$ \\
\hline LVEDD 60-70 mm & $0.76(0.51-1.12)$ & $0.75(0.50-1.10)$ & $0.75(0.51-1.10)$ \\
\hline LVEDD $>70 \mathrm{~mm}$ & $0.82(0.55-1.23)$ & $0.84(0.56-1.24)$ & $0.83(0.56-1.23)$ \\
\hline Diabetes mellitus & $1.24(0.95-1.62)$ & $1.27(0.97-1.66)$ & $1.22(0.93-1.59)$ \\
\hline Creatinine $80-100 \mu \mathrm{mol} / \mathrm{L}$ & $1.10(0.73-1.64)$ & $1.07(0.72-1.61)$ & $1.10(0.73-1.65)$ \\
\hline Creatinine $101-120 \mu \mathrm{mol} / \mathrm{L}$ & $1.07(0.72-1.60)$ & $0.99(0.64-1.52)$ & $1.06(0.69-1.63)$ \\
\hline Creatinine $>120 \mu \mathrm{mol} / \mathrm{L}$ & $1.30(0.87-1.96)$ & $1.25(0.83-1.88)$ & $1.32(0.88-1.98)$ \\
\hline
\end{tabular}

${ }^{*} \mathrm{p}<0.05 ;{ }^{* *} \mathrm{p}<0.01 ; \mathrm{Cl}$ - confidence interval; HR - hazard ratio; other abbreviations - see Table 2

Table 4. Multivariate Cox regression models for the combined endpoint of all-cause mortality and heart failure hospitalisations in patients with and without left bundle branch block (LBBB) according to the Perrin and Strauss definitions and the European Society of Cardiology (ESC) guidelines QRS morphology criteria for cardiac resynchronisation therapy (CRT) class I indications with regard to potential confounding variables

\begin{tabular}{|c|c|c|c|}
\hline & \multicolumn{3}{|c|}{ HR $(95 \% \mathrm{Cl})$} \\
\hline & Perrin & Strauss & ESC CRT guidelines \\
\hline LBBB & $0.58^{* *}(0.43-0.79)$ & $0.50 * *(0.38-0.66)$ & $0.68 *(0.53-0.88)$ \\
\hline Age 60-70 years & $0.98(0.71-1.37)$ & $0.83(0.59-1.17)$ & $0.95(0.68-1.33)$ \\
\hline Age $>70$ years & $1.07(0.77-1.48)$ & $0.92(0.66-1.28)$ & $1.02(0.74-1.41)$ \\
\hline Male sex & $0.94(0.68-1.30)$ & $0.93(0.67-1.29)$ & $0.95(0.68-1.31)$ \\
\hline Ischaemic aetiology & $1.15(0.88-1.49)$ & $1.12(0.86-1.46)$ & $1.16(0.89-1.51)$ \\
\hline NYHA III & $1.52(0.86-2.68)$ & $1.62(0.92-2.85)$ & $1.58(0.90-2.79)$ \\
\hline NYHA IV & $2.90 * *(1.56-5.40)$ & $3.18^{* *}(1.71-5.91)$ & $3.11 * *(1.67-5.79)$ \\
\hline Permanent AF & $1.03(0.79-1.34)$ & $0.92(0.70-1.21)$ & $0.97(0.74-1.27)$ \\
\hline LVEF $20 \%-30 \%$ & $0.79(0.61-1.04)$ & $0.80(0.62-1.05)$ & $0.81(0.62-1.05)$ \\
\hline LVEF $>30 \%$ & $0.68(0.47-1.00)$ & $0.64 *(0.44-0.94)$ & $0.65^{*}(0.45-0.96)$ \\
\hline LVEDD 60-70 mm & $0.81(0.58-1.15)$ & $0.81(0.57-1.15)$ & $0.81(0.57-1.14)$ \\
\hline LVEDD > $70 \mathrm{~mm}$ & $0.86(0.60-1.22)$ & $0.88(0.61-1.25)$ & $0.87(0.61-1.24)$ \\
\hline Diabetes mellitus & $1.34 *(1.06-1.70)$ & $1.35 *(1.07-1.71)$ & $1.32 *(1.04-1.67)$ \\
\hline Creatinine $80-100 \mu \mathrm{mol} / \mathrm{L}$ & $1.04(0.73-1.48)$ & $1.05(0.74-1.49)$ & $1.07(0.75-1.52)$ \\
\hline Creatinine $100-120 \mu \mathrm{mol} / \mathrm{L}$ & $1.05(0.72-1.53)$ & $0.99(0.68-1.44)$ & $1.05(0.72-1.54)$ \\
\hline Creatinine $>120 \mu \mathrm{mol} / \mathrm{L}$ & $1.34(0.94-1.93)$ & $1.32(0.92-1.90)$ & $1.37(0.95-1.97)$ \\
\hline
\end{tabular}

${ }^{*} p<0.05 ;{ }^{* *} p<0.01$; abbreviations - see Tables 2 and 3 
patients with an $\mathrm{R}$ wave $\geq 1 \mathrm{~mm}$ in $\mathrm{V}_{1}$, despite other features pointing to a true LBBB (notches, QRS duration), results in the inclusion of good CRT responders into the non-LBBB group and hence the small difference in mortality between LBBB and non-LBBB subgroups - as was observed in the current study.

We have previously assessed the prognostic value of the Strauss definition and compared it with three other LBBB definitions [13]. That study showed the superiority of the Strauss definition over the conventional, Marriott and WHO/AHA definitions of LBBB [13]. However, in that study we did not assess the new Perrin definition and we failed to compare these definitions with the QRS morphology fulfilling the highest level of indications for CRT according to the recognised guidelines. Some studies suggest that QRS duration is more important than QRS morphology (i.e. LBBB vs. non-LBBB) [11]. The CRT guidelines on QRS morphology criteria for class I/ /level of evidence A potentially combine the "best of both worlds" - both LBBB morphology criteria and QRS duration criteria. However, such QRS morphology criteria were never compared against new, stricter LBBB definitions. These issues were addressed in the current study: the completion of the ESC CRT guideline QRS criteria was found to be inferior to fulfilment of the refined LBBB morphology criteria alone (Strauss definition). This suggests that the QRS duration is less important than the diagnosis of a complete LBBB. Similarly, compliance with the Perrin "true LBBB" criteria did not surpass the completion of the "complete LBBB" definition by Strauss. The only other study that assessed the "true LBBB" definition was the analysis performed on the patients included in the PREDICT study by Perrin et al. [18]. In that analysis, only a three-month echocardiographic response was assessed; therefore, it is not directly comparable with the current study, which, for the first time, assessed the prognostic value of these criteria with regard to the "hard endpoint" of mortality and morbidity, had a much longer follow-up, and was based on a much larger cohort (552 vs. 40 patients). However, the percentage of LBBB/NIVCD patients in whom "true LBBB" criteria were fulfilled was similar in both studies: $30 \%$ in the study by Perrin et al. [18] vs. $34 \%$ in the current study. This points to a similar ECG assessment methodology, the exclusive nature of these criteria, and the comparability of the studied groups.

The strengths of our study include the large sample size of the analysed patients, the availability of complete medical data and ECG recordings for almost all participants, and the long follow-up period. The sizable number of patients meeting the primary endpoint, with lack of cases whose status could not be determined, ensures the reliability of mortality data. The limitations are typical for any type of retrospective, longitudinal, single-centre cohort study. There is a possibility of the presence of unmeasured confounding variables not accounted for during this investigation. The single-centre design could lead to the possibility of the introduction of treatment bias, which could have influenced the outcome of the CRT. The patient cohort evaluated in the present study were comparable to cohorts in other large studies assessing the outcomes of CRT, increasing the reliability of the presented results. Devices were implanted using standard approaches by several experienced electrophysiologists and the mortality rates were within the expected range for patients predominantly in New York Heart Association class III.

In conclusion, it seems that the "true LBBB" criteria proposed by Perrin et al. [18] are not well-suited for the selection of CRT candidates, due to the fact they do not reflect the presence of a true LBBB. The results of this study add further evidence to the fact that the new LBBB definition proposed by Strauss is superior to other QRS morphological criteria in identifying good CRT responders. This is possibly related to better identification of patients with complete LBBB or a significant delay of the left ventricular free wall that is correctable with biventricular pacing.

\section{Conflict of interest: none declared}

\section{References}

1. Jastrzebski M, Baranchuk A, Fijorek K, et al. Cardiac resynchronization therapy-induced acute shortening of QRS duration predicts long-term mortality only in patients with left bundle branch block. Europace. 2019; 21(2): 281-289, doi: 10.1093/europace/euy254, indexed in Pubmed: 30403774

2. Kisiel R, Fijorek K, Sondej T, et al. Risk stratification in patients with cardiac resynchronisation therapy: the AL-FINE CRT risk score. Kardiol Pol. 2018; 76(10): 1441-1449, doi: 10.5603/KP.a2018.0152, indexed in Pubmed: 30251245.

3. Kisiel R, Fijorek K, Moskal P, et al. New ECG markers for predicting long-term mortality and morbidity in patients receiving cardiac resynchronization therapy. J Electrocardiol. 2018; 51(4): 637-644, doi: 10.1016/j.jelectrocard.2018.04.007, indexed in Pubmed: 29997004

4. Jin H, Yang S, Hua W, et al. Significant mitral regurgitation as a predictor of long-term prognosis in patients receiving cardiac resynchronisation therapy. Kardiol Pol. 2018; 76(6): 987-992, doi: 10.5603/KP.a2018.0035, indexed in Pubmed: 29399763.

5. Płońska-Gościniak E, Kasprzak JD, Kukulski T, et al. Role of lowdose dobutamine echocardiography in predicting response to biventricular pacing. Results from the multicenter Viability in Cardiac Resynchronisation Therapy (ViaCRT) study. Pol Arch Med Wewn. 2016; 126(12): 989-994, doi: 10.20452/pamw.3715, indexed in Pubmed: 27958262.

6. Pres D, Niedziela J, Kurek A, et al. In-hospital and long-term prognosis in patients after implantation of implantable cardioverter-defibrillators and cardiac resynchronization therapy: 10-year results of the SILCARD registry. Pol Arch Intern Med. 2018; 128(10): 580-586, doi: 10.20452/pamw.4332, indexed in Pubmed: 30215623.

7. Sipahi I, Chou JC, Hyden M, et al. Effect of QRS morphology on clinical event reduction with cardiac resynchronization therapy: meta-analysis of randomized controlled trials. Am Heart J. 2012; 163(2): 260-7.e3, doi: 10.1016/j.ahj.2011.11.014, indexed in Pubmed: 22305845.

8. Khidir MJH, Delgado V, Ajmone Marsan N, et al. QRS duration versus morphology and survival after cardiac resynchronization therapy. ESC Heart Fail. 2017; 4(1): 23-30, doi: 10.1002/ehf2.12122, indexed in Pubmed: 28217309.

9. Jastrzebski M, Wilinski J, Fijorek K, et al. Mortality and morbidity in cardiac resynchronization patients: impact of lead position, paced left ventricular QRS morphology and other characteristics on long-term outcome. Europace. 2013; 15(2): 258-265, doi: 10.1093/europace/eus340, indexed in Pubmed: 23047489. 
10. Zareba W, Klein H, Cygankiewicz I, et al. Effectiveness of Cardiac Resynchronization Therapy by QRS Morphology in the Multicenter Automatic Defibrillator Implantation Trial-Cardiac Resynchronization Therapy (MADIT-CRT). Circulation. 2011; 123(10): 1061-1072, doi: 10.1161/CIRCULATIONAHA.110.960898, indexed in Pubmed: 21357819.

11. Cleland JG, Abraham WT, Linde C, et al. An individual patient meta-analysis of five randomized trials assessing the effects of cardiac resynchronization therapy on morbidity and mortality in patients with symptomatic heart failure. Eur Heart J. 2013; 34(46): 3547-3556, doi: 10.1093/eurheartj/eht290, indexed in Pubmed: 23900696.

12. Kronborg MB, Nielsen JC, Mortensen PT. Electrocardiographic patterns and long-term clinical outcome in cardiac resynchronization therapy. Europace. 2010; 12(2): 216-222, doi: 10.1093/europace/eup364, indexed in Pubmed: 19915182.

13. Jastrzębski M, Kukla P, Kisiel R, et al. Comparison of four LBBB definitions for predicting mortality in patients receiving cardiac resynchronization therapy. Ann Noninvasive Electrocardiol. 2018; 23(5): e12563, doi: 10.1111/anec.12563, indexed in Pubmed: 29806716.

14. Caputo ML, van Stipdonk A, Illner A, et al. The definition of left bundle branch block influences the response to cardiac resynchronization therapy. Int J Cardiol. 2018; 269: 165-169, doi: 10.1016/j.ijcard.2018.07.060, indexed in Pubmed: 30025653.

15. Strauss DG. Importance of defining left bundle branch block J Electrocardiol. 2012; 45(5): 505-507, doi: 10.1016/j.jelectrocard.2012.06.023, indexed in Pubmed: 22920786.

16. Strauss DG, Selvester RH, Wagner GS. Defining left bundle branch block in the era of cardiac resynchronization therapy. Am J Cardiol. 2011; 107(6): 927-934, doi: 10.1016/j.amjcard.2010.11.010, indexed in Pubmed: 21376930.

17. Padanilam BJ, Morris KE, Olson JA, et al. The surface electrocardiogram predicts risk of heart block during right heart catheterization in patients with preexisting left bundle branch block: implications for the definition of complete left bundle branch block. J Cardiovasc Electrophysiol. 2010; 21(7): 781-785, doi: 10.1111/j.1540-8167.2009.01714.x, indexed in Pubmed: 20132380.
18. Perrin MJ, Green MS, Redpath CJ, et al. Greater response to cardiac resynchronization therapy in patients with true complete left bundle branch block: a PREDICT substudy. Europace. 2012; 14(5): 690-695, doi: 10.1093/europace/eur381, indexed in Pubmed: 22170897.

19. Mascioli G, Padeletti L, Sassone B, et al. Electrocardiographic criteria of true left bundle branch block: a simple sign to predict a better clinical and instrumental response to CRT. Pacing Clin Electrophysiol. 2012; 35(8): 927-934, doi: 10.1111/j.1540-8159. 2012.03427.x, indexed in Pubmed: 22651702.

20. Tian Y, Zhang P, Li X, et al. True complete left bundle branch block morphology strongly predicts good response to cardiac resynchronization therapy. Europace. 2013; 15(10): 1499-1506, doi: 10.1093/europace/eut049, indexed in Pubmed: 23468351.

21. Surawicz B, Childers R, Deal BJ, et al. AHA/ACCF/HRS recommendations for the standardization and interpretation of the electrocardiogram: part III: intraventricular conduction disturbances: a scientific statement from the American Heart Association Electrocardiography and Arrhythmias Committee, Council on Clinical Cardiology; the American College of Cardiology Foundation; and the Heart Rhythm Society: endorsed by the International Society for Computerized Electrocardiology. Circulation. 2009; 119(10): e235-e240, doi: 10.1161/CIRCULATIONAHA.108.191095, indexed in Pubmed: 19228822.

22. Willems JL, Robles de Medina EO, Bernard R, et al. Criteria for intraventricular conduction disturbances and pre-excitation. World Health Organizational/International Society and Federation for Cardiology Task Force Ad Hoc. J Am Coll Cardiol. 1985; 5(6): 1261-1275, indexed in Pubmed: 3889097.

23. Brignole M, Auricchio A, Baron-Esquivias G, et al. 2013 ESC Guidelines on cardiac pacing and cardiac resynchronization therapy: the Task Force on cardiac pacing and resynchronization therapy of the European Society of Cardiology (ESC). Developed in collaboration with the European Heart Rhythm Association (EHRA). Eur Heart J. 2013; 34(29): 2281-2329, doi: 10.1093/eurheartj/eht150, indexed in Pubmed: 23801822.

24. Wellens HJ. Is the left bundle branch really blocked when suggested by the electrocardiogram? Europace. 2012; 14(5): 619-620, doi: 10.1093/europace/eur401, indexed in Pubmed: 22266052 .

Cite this article as: Kisiel R, Fijorek K, Moskal P, et al. True left bundle branch block and long-term mortality in cardiac resynchronisation therapy patients. Kardiol Pol. 2019; 77(3): 371-379, doi: 10.5603/KP.a2019.0032.

\section{WHAT IS NEW?}

For the first time, an evaluation of the strict left bundle branch block (LBBB) criteria recently proposed by Perrin et al. was performed in a sizable cohort of patients with cardiac resynchronisation therapy (CRT). To our best knowledge, this is the first study assessing the Perrin LBBB definition with regard to the long-term mortality and morbidity and the only "head-to-head" comparison of this definition with the other increasingly recognised strict LBBB criteria proposed by Strauss. The study also offers the first comparison of the prognostic impact of QRS morphology that combines both bundle branch criteria and extra QRS duration requirements as proposed by the European Society of Cardiology CRT guideline indications (class I, level of evidence A), versus both Perrin and Strauss strict LBBB definitions. The major practical application of these novel findings is that the Perrin LBBB definition is not advantageous when compared to the Strauss LBBB definition; our data do not support the notion that it should be preferred for CRT candidate selection. The other practical conclusion is that the presence of a substantial $\mathrm{R}$ wave in $\mathrm{V}_{1}$ probably should not be considered as a clue that LBBB is not complete. 